\title{
Analyse de données sur les ventes lors d'une intervention axée sur un dépanneur santé de Toronto : le projet FRESH sur l'environnement de la vente d'aliments au détail comme déterminant de la santé
}

Leia M. Minaker, Ph. D. (1); Meghan Lynch, Ph. D. (2); Brian E. Cook, Ph. D. (3); Catherine L. Mah, M.D., Ph. D. (2,4)

Cet article a fait l'objet d'une évaluation par les pairs.

\section{Résumé}

Introduction : Les interventions en santé de la population dans le secteur de l'alimentation de détail, comme celles réalisées dans les dépanneurs, visent à transformer le type de signaux envoyés aux consommateurs afin qu'ils choisissent des aliments plus sains. Peu de recherches abordent les aspects financiers des interventions réalisées dans le milieu de la vente au détail, en particulier les mesures de résultat comme les ventes en magasin, pourtant au centre du processus décisionnel de la vente au détail. Cette étude examine l'évolution des ventes en magasin et les ventes par catégories de produits dans le cadre d'une intervention axée sur un dépanneur santé situé dans un quartier à faible revenu de Toronto (Ontario).

Méthodologie : Les données sur les ventes effectuées entre août 2014 et avril 2015 ont été regroupées par catégories de produits et par jour. Nous avons utilisé des tableaux croisés dynamiques Excel afin de résumer et de présenter visuellement les données sur ces ventes. Nous avons mené des tests $t$ afin d'étudier les différences au niveau des ventes de chaque catégorie de produits en fonction des jours de vente " de pointe » (jours d'affluence) par rapport aux autres jours de vente.

Résultats : Les ventes globales du magasin culminaient les derniers jours de chaque mois, soit à la période à laquelle les prestations d'assistance sociale sont versées. La hausse des revenus lors des jours de pointe était principalement imputable aux ventes de laissez-passer de transports en commun. La moyenne des ventes de collations non nutritives et de cigarettes était légèrement plus élevée lors des jours de pointe par rapport aux autres jours. Les stratégies novatrices employées pour augmenter les ventes de fruits et légumes frais ont semblé accroître considérablement les revenus générés par ces catégories de produits.

Conclusion : Les données sur les ventes en magasin constituent un paramètre important de mesure du succès des interventions en environnement alimentaire. De plus, les prises de décision des détaillants fondé sur ces données peuvent se révéler déterminantes lors de l'adaptation des interventions. Les responsables des recherches et des interventions futures devraient envisager d'établir des partenariats et d'utiliser d'autres indicateurs de rendement lors des interventions ciblant l'environnement de la vente au détail d'aliments dans divers contextes canadiens.

Mots-clés : secteur de l'alimentation de détail, intervention en santé de la population, données sur les ventes, dépanneurs
Points saillants

- Les praticiens de la santé publique ayant un intérêt pour les interventions dans le secteur de l'alimentation au détail peuvent utiliser les données relatives aux ventes pour élaborer des évaluations globales.

- Les données relatives aux ventes peuvent être utilisées pour adapter des interventions axées sur des dépanneurs santé au contexte local (en offrant, par exemple, des promotions les jours de pointe des ventes).

- L'utilisation des données relatives aux ventes est importante pour la recherche et pour les propriétaires de commerces de détail, ces derniers pouvant utiliser ces indicateurs pour adapter leurs pratiques commerciales.

\section{Introduction}

Les interventions dans le secteur de l'alimentation de détail visant les commerces (p. ex. celles axées sur les épiceries et les dépanneurs) sont de plus en plus reconnues comme des interventions en santé publique importantes pour l'amélioration de la valeur nutritionnelle des aliments achetés ${ }^{1-4}$. L'objectif de ces interventions est d'encourager des comportements alimentaires plus sains en améliorant l'accessibilité et la disponibilité en choix alimentaires nutritifs et abordables dans l'environnement nutritionnel du

Rattachement des auteurs :

1. École de planification, Université de Waterloo, Waterloo (Ontario), Canada

2. École de santé publique Dalla Lana, Université de Toronto, Toronto (Ontario), Canada

3. Stratégie alimentaire de Toronto, Bureau de santé publique de Toronto, Toronto (Ontario), Canada

4. Faculté de la santé, Dalhousie University, Halifax (Nouvelle-Écosse), Canada

Correspondance : Leia M. Minaker, École de planification, Université de Waterloo; 200, av. University Ouest, Waterloo (Ontario) N2L 3G1; tél. : 519-888-4567, poste 35615; courriel : Iminaker@uwaterloo.ca 
consommateur et au sein de la collectivité $^{5,6}$. Pratiquer dans les magasins d'alimentation des interventions favorisant une saine alimentation est particulièrement important dans la mesure où pour chaque dollar réservé à l'alimentation dans un ménage, plus de 70 cents y sont dépensés plutôt que dans les restaurants ${ }^{7}$.

Les données sur les ventes d'aliments ont été proposées afin de mesurer les comportements alimentaires, car elles sont utiles, objectives, rentables et discrètes et n'exercent aucun fardeau sur des individus $^{8}$. Nous avons utilisé des données sur les ventes en magasin (données directes de lecteurs optiques d'étiquettes, ensembles de données commerciales accessibles et reçus de caisse d'épicerie) ainsi qu'un certain nombre d'études récentes portant sur de petits commerces ${ }^{13-17}$ afin d'observer l'efficacité des interventions dans divers types de magasins d'alimentation au détail ${ }^{9-12}$. Pris ensemble, les résultats de ces études semblent indiquer que des données sur les ventes pourraient servir à établir des stratégies d'intervention ciblant l'alimentation au détail. Par exemple, Foster et ses collègues ${ }^{9}$ ont constaté que des stratégies à faible coût axées sur l'amélioration de l'accessibilité des produits et sur les stratégies de placement conduisaient à accroître les ventes de certaines catégories d'aliments et de boissons (lait, eau, repas surgelés) au détriment d'autres catégories (boissons gazeuses régulières et diète, céréales).

Un examen systématique récent de l'efficacité des interventions en supermarché visant à améliorer la valeur nutritive des aliments achetés par les consommateurs a révélé qu'aucune des 49 études pertinentes recensées ne faisait état des répercussions économiques ou financières des interventions sur le détaillant ${ }^{4}$. En 2012, un examen descriptif des stratégies marketing d'épiceries visant à améliorer l'alimentation a révélé que seulement 5 des 125 études évaluées par des pairs avaient utilisé des données sur les ventes pour mesurer les répercussions de diverses stratégies ${ }^{3}$. D'après un autre examen de 2012 portant sur les répercussions d'interventions axées sur la vente au détail dans de petits magasins d'alimentation, aucune analyse de données sur les ventes n'avait été réalisée dans la majorité des études (11 sur 16) ${ }^{2}$. Seules quelques études ont utilisé des données sur les ventes comme principal critère de résultat pour évaluer le rendement de la vente au détail des commerces ${ }^{18}$. Plusieurs raisons expliquent que les recherches antérieures n'aient pas eu recours à des données sur les ventes, notamment la nature potentiellement sensible des données et la mauvaise qualité de ces dernières, attribuable à l'erreur humaine ou à des obstacles technologiques. La rareté des résultats publiés sur les ventes n'en demeure pas moins une lacune importante dans ce domaine de recherche parce que, même si des interventions bien conçues ciblant le secteur de l'alimentation au détail améliorent les comportements alimentaires de la population, leurs effets sur la population ne pourront être maintenus que si elles satisfont aux objectifs économiques des détaillants.

Compte tenu de l'importance des données économiques dans le processus décisionnel en vente au détail, il est crucial de considérer les données sur les ventes comme un paramètre important de l'évaluation des interventions dans le secteur de l'alimentation au détail, pour assurer la mise en œuvre comme la durabilité des interventions ${ }^{4}$. Dans ce secteur, la capacité des petits magasins à prendre des décisions fondées sur les données est restreinte. L'évaluation des ventes peut contribuer à améliorer la promotion des interventions axées sur la vente au détail d'aliments sains auprès de divers détaillants en alimentation ${ }^{19}$, d'autant plus que les propriétaires de commerce sont soucieux des pertes potentielles de revenus ${ }^{20,21}$. De fait, l'absence de prise en compte des données sur les ventes dans le cadre d'études antérieures pourrait constituer un obstacle à ce que d'autres commerces adoptent des stratégies d'intervention en matière d'alimentation saine. Ce type d'information est important non seulement pour les détaillants, mais également pour les législateurs travaillant à l'élaboration de politiques à l'appui d'un environnement alimentaire sain dans les provinces et territoires du Canada $^{22}$.

L'objectif de notre étude était de caractériser l'évolution des ventes en magasin dans le cadre d'une intervention axée sur la vente au détail d'aliments sains réalisée dans un quartier urbain à faible revenu. Nous avons examiné les ventes de boissons et d'aliments ainsi que les ventes de produits non alimentaires relevant de plusieurs catégories. Dans cet article, nous présentons certaines données liées à la vente de produits non alimentaires afin de donner un aperçu des risques éventuels auxquels les petits commerces de détail s'exposent lorsqu'ils mettent en œuvre une stratégie d'intervention axée sur la vente au détail d'aliments sains, ainsi que des opportunités qui leur sont offertes.

\section{Méthodologie}

L'étude FRESH (Food Retail Environments Shaping Health) sur les milieux de la vente d'aliments au détail comme déterminants de la santé a été financée par l'Agence de la santé publique du Canada et menée dans le cadre de la Stratégie alimentaire de Toronto (une initiative du Bureau de santé publique de Toronto). Les données ont été recueillies d'août 2014 à avril 2015. L'étude FRESH a été réalisée au moyen de méthodes combinées, afin d'évaluer l'alimentation sur le plan individuel et les effets sur la sécurité alimentaire d'une intervention pilote axée sur un dépanneur santé et un marché mobile d'aliments santé (un autobus de ville modifié distribuant et vendant des fruits et des légumes frais dans des quartiers ayant difficilement accès à des épiceries) situés dans deux quartiers à faible revenu de Toronto (Ontario). Cet article présente les données sur les ventes du dépanneur qui a participé à l'intervention visant les dépanneurs santé. Malheureusement, les données sur les ventes du marché mobile d'aliments santé se sont révélées de piètre qualité et n’ont donc pu être présentées.

\section{Contexte}

Le dépanneur ayant fait l'objet d'une intervention se situait dans le quartier 43 de l'arrondissement Scarborough-Est à Toronto (Ontario). Il s'agit d'un quartier où $42 \%$ des habitants vivent dans des immeubles d'habitation comprenant plus de cinq étages, où $52 \%$ des habitants sont nés hors Canada, où $57 \%$ des habitants ont pour langue maternelle l'anglais et où le revenu annuel moyen des ménages est inférieur d'environ 20000 \$ au revenu annuel moyen de l'ensemble de la ville de Toronto $^{23,24}$. Le dépanneur ayant fait l'objet d'une intervention se trouvait au cœur de Scarborough-Est, au rez-de-chaussée d'un complexe de tours d'habitations collectives. L'endroit a été choisi conjointement par l'équipe de la Stratégie alimentaire de Toronto et l'East Scarborough Storefront, un organisme communautaire visant à aider la population et à bâtir une communauté à Scarborough-Est. Nous avons demandé à des commerçants de participer au projet, et ils ont pris part activement à 
toutes les décisions concernant l'intervention et à la collecte des données sur les ventes. Des données inédites provenant de l'étude FRESH à grande échelle (qui comprend des enquêtes menées auprès de 199 consommateurs d'aliments de base vivant dans les tours d'habitation ayant fait l'objet de l'intervention) ont révélé que parmi les habitants de l'ensemble des tours d'habitations collectives où se situait le commerce, 83,3\% étaient nés à l'extérieur du Canada, 69 \% avaient au moins un enfant et $78 \%$ avaient un revenu familial annuel inférieur à 30000 \$.

\section{Intervention}

La transformation du dépanneur servant de modèle au projet a consisté en un processus itératif englobant (1) les bases commerciales, en particulier l'approvisionnement alimentaire, les infrastructures et l'analyse des ventes, (2) le service à la clientèle et la mobilisation des clients et (3) diverses stratégies de marchandisage. Les principaux objectifs de l'intervention étaient d'améliorer les relations avec les fournisseurs et le marchandisage afin d'accroître l'accessibilité des boissons et aliments nutritifs et leur importance. Dans le cadre de cette intervention, ce qui a considéré comme «boissons et aliments nutritifs » correspondait aux recommandations du Guide alimentaire canadien. En collaboration avec les diététistes en santé publique du Bureau de santé publique de Toronto et les propriétaires du commerce, nous avons sélectionné précisément les boissons et aliments nutritifs à mettre en vente (p. ex. fruits et légumes entiers frais, eau, maïs soufflé, barres de céréales faibles en sucre, collations de légumes). Nous avons présenté et promus ces aliments nutritifs pendant la période allouée à l'intervention. Ces produits nutritifs ont été offerts à des prix concurrentiels avec ceux de produits comparables vendus dans le supermarché à bas prix proche (à environ $1 \mathrm{~km}$ ). Le dépanneur a été choisi en décembre 2013, les changements apportés à l'infrastructure pour la vente de denrées fraîches, comme l'achat et l'installation de nouveaux réfrigérateurs ainsi que la modification des rayonnages, ont été effectués entre juin 2014 et février 2015 et les produits frais ont été proposés à partir de juin 2014.

Afin d'augmenter l'approvisionnement annuel du commerce en fruits et légumes frais, l'équipe de la Stratégie alimentaire de Toronto a mis les propriétaires du dépanneur en contact avec un grossiste torontois de fruits et de légumes frais des environs. L'équipe de la Stratégie alimentaire de Toronto a aidé les propriétaires à ouvrir un compte et leur a appris à passer leurs commandes. Les propriétaires se sont également procuré des fruits et des légumes dans un supermarché asiatique proche. Des membres de l'équipe de recherche se sont rendus au dépanneur d'une à trois fois par semaine durant l'intervention afin d'offrir un soutien continu aux commerçants, en particulier pour effectuer la collecte de données sur les ventes au moyen du système de point de vente (décrit ci-dessous).

Il est à noter pour cette étude que la compagnie de location immobilière propriétaire de la tour d'habitations offrait un programme permanent de collations gratuites chaque jour d'école pour tous les enfants de la tour d'habitations. Chaque semaine, les bailleurs achetaient des denrées fraîches pour ce programme de collation dans un club-entrepôt afin de les distribuer aux élèves. En novembre 2014, les propriétaires du dépanneur ont réussi à négocier une entente avec les bailleurs afin de leur fournir des produits frais au même prix pour le programme de collations.

Plusieurs défis à la mise en œuvre de l'intervention sur la durée sont survenus : améliorer les activités commerciales générales, sensibiliser les propriétaires du commerce à prendre en compte la qualité des données, renforcer la capacité décisionnelle en matière de vente au détail axée sur les données, élaborer des stratégies de marchandisage, établir des liens avec la communauté et remédier à la complexité des relations entre les propriétaires du dépanneur et les clients réguliers. Ces défis sont similaires à ceux observés dans d'autres contextes d'intervention concernant des dépanneurs santée ${ }^{20}$.

\section{Mesures}

Nous avons recueilli des données sur les ventes au moyen d'un système de point de vente mis en œuvre en janvier 2014. Ce système a permis de recenser les dates et les heures des ventes, les codes CUP, les catégories de produits déterminées par les chercheurs (p. ex. boissons sucrées, eau embouteillée, friandises, fruits, légumes, billets de loterie, cigarettes, etc.) ainsi que la quantité et les prix des articles achetés. Une compagnie spécialisée dans le marketing numérique pratiqué dans les dépanneurs a soutenu le projet en fournissant gratuitement l'équipement, l'installation et la maintenance du point de vente ainsi que la formation sur son fonctionnement. Elle a également fait le suivi de l'efficacité de la publicité effectuée dans le dépanneur et a consenti à faire de la publicité seulement pour les boissons et les aliments nutritifs pendant toute la durée du projet. L'équipe de recherche pouvait demander régulièrement un registre à jour des données sur les ventes au fournisseur du point de vente tout au long du projet. Le système de point de vente a été installé en janvier 2014, mais plusieurs mois se sont écoulés avant l'obtention de données fiables et avant que les propriétaires du dépanneur aient atteint une capacité efficace de gestion de données et maîtrisé l'utilisation du système. Par conséquent, nous présentons ici uniquement les données de qualité obtenues entre août 2014 et avril 2015 (le dernier mois au cours duquel les données étaient accessibles). De plus, nous avons restreint l'objet de nos analyses au chiffre d'affaires (le total des ventes du commerce issu des ventes d'articles de détail) sans tenir compte des profits (revenus moins les dépenses), étant donné que les données sur les dépenses n’ont pas été recueillies.

\section{Analyse des données}

Nous avons regroupé les données sur les ventes par catégorie de produits et par jour afin d'étudier l'évolution des ventes en magasin. Nous avons eu recours à des tableaux croisés dynamiques Excel afin d’organiser et de synthétiser les données. Nous avons également créé des représentations visuelles des données sur les ventes à l'aide des fonctions graphiques de Microsoft Excel.

En premier lieu, nous avons résumé les ventes totales du magasin par jour, puis consigné à l'aide de graphiques les données recueillies au fil du temps afin d'analyser visuellement la constance des ventes en magasin au cours de la période étudiée. Selon nos premières constatations, les ventes en magasin ont semblé monter en flèche lors de certains jours au cours du mois, que nous désignerons désormais par « jours de ventes de pointe » dans cet article. Une recherche plus approfondie a révélé que les jours de ventes de pointe avaient toujours lieu le jour suivant le versement des prestations d'assistance sociale. Nous avons analysé les ventes quotidiennes de produits dans différentes 
catégories lors des jours de ventes de pointe par rapport aux autres jours de ventes et avons créé des tests $t$ bilatéraux au moyen de variances inégales afin de voir s'il y avait des différences significatives dans les ventes de chaque catégorie de produits lors des jours de pointe par rapport aux autres jours, avec une valeur statistiquement significative $p<0,05$.

En second lieu, nous avons représenté graphiquement les revenus mensuels générés par les différentes catégories de produit au fil du temps. La vente de fruits et légumes (une des principales composantes de l'intervention) a également été analysée visuellement dans le temps. Étant donné l'entente conclue, en novembre 2014, entre les propriétaires du dépanneur et les bailleurs au sujet de l'approvisionnement en produits frais dans le cadre du programme de collations sans frais (décrit plus haut), nous présentons les données sur les ventes de fruits et légumes avec et sans les ventes liées au programme de collations, dans le but de fournir une représentation exacte des ventes de fruits et légumes aux clients réguliers.

\section{Résultats}

La figure 1 illustre les ventes quotidiennes totales (à l'exception des ventes de loterie) réalisées dans le cadre de l'intervention pilote axée sur le dépanneur entre août 2014 et avril 2015. La figure 2 présente la moyenne des ventes quotidiennes de différentes catégories de produits effectuées durant les jours de ventes de pointe et les autres jours de ventes. Lors des jours de ventes de pointe et les autres jours, les billets et laissez-passer de transports en commun, les billets de loterie et les cigarettes constituaient les ventes globales les plus élevées. Lors des jours de ventes de pointe, la moyenne des ventes quotidiennes de cigarettes était légèrement plus élevée par rapport aux autres jours $(175,73 \$$ contre $130,09 \$ ; p=0,084)$. La moyenne des ventes de collations et de boissons non nutritives était aussi légèrement plus élevée lors des jours de pointe $(85,04 \$$ pour les collations et 74,34 $\$$ pour les boissons par jour) comparativement aux autres jours $(61,67 \$$ pour les collations $[p=0,078]$ et $56,39 \$$ pour les boissons [ $p=0,145]$ par jour). Les ventes de loteries $(p=0,023)$ et les ventes de billets pour les transports en commun $(p<0,0001)$ étaient significativement plus élevées les jours de pointe.

La figure 3 présente les revenus mensuels générés au fil du temps par la vente de produits appartenant à quatre catégories : des denrées fraîches, des collations (comprenant des friandises, du chocolat, des croustilles et d'autres collations salées et collations faites de viande), des boissons sucrées et des cigarettes. Durant toute la durée de l'intervention, les revenus générés par la vente de cigarettes étaient élevés. Ceux tirés de la vente de denrées fraîches allaient de 165 \$ en août 2014 à plus de 1000 \$ par mois pour la période allant de janvier à avril 2015. Les revenus générés par la vente de denrées fraîches dépassaient les revenus tirés de la vente de boissons sucrées et de collations en janvier 2015, mais ils étaient légèrement plus bas que ceux provenant de la vente de boissons et de collations en mars et en avril 2015.

Enfin, les ventes brutes mensuelles de fruits et légumes sont présentées dans la figure 4 en fonction du revenu global et selon le revenu généré par les ventes aux clients (c'est-à-dire sans les ventes de fruits et légumes aux bailleurs). En moyenne, le dépanneur a généré chaque mois des revenus d'environ 935 \$ grâce à la vente de produits frais durant les six mois pendant lesquels les bailleurs ont acheté des fruits au commerce visé par l'intervention.

\section{Analyse}

Cette étude exploratoire décrit les caractéristiques de données sur les ventes d'un

FIGURE 1

Total des ventes quotidiennes d'un dépanneur, à l'exception des ventes de loteries, au cours d'une intervention ciblant un dépanneur santé de Toronto (Ontario), août 2014 à avril 2015

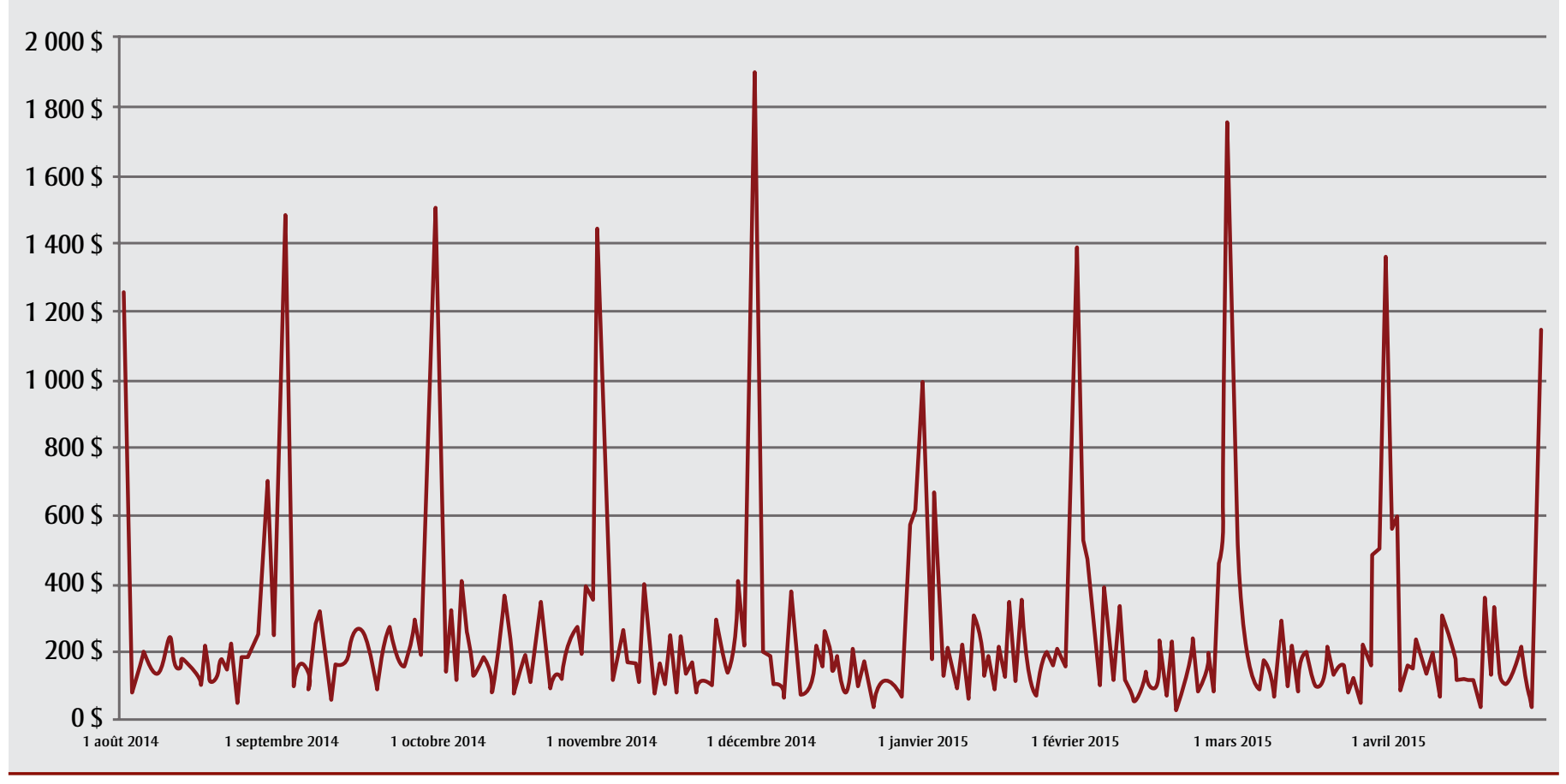


FIGURE 2

Moyenne des ventes quotidiennes issues de différentes catégories de produits lors des jours de pointe (jours de ventes les plus élevées) et des autres jours au cours d'une intervention ciblant un dépanneur santé de Toronto (Ontario), août 2014 à avril 2015

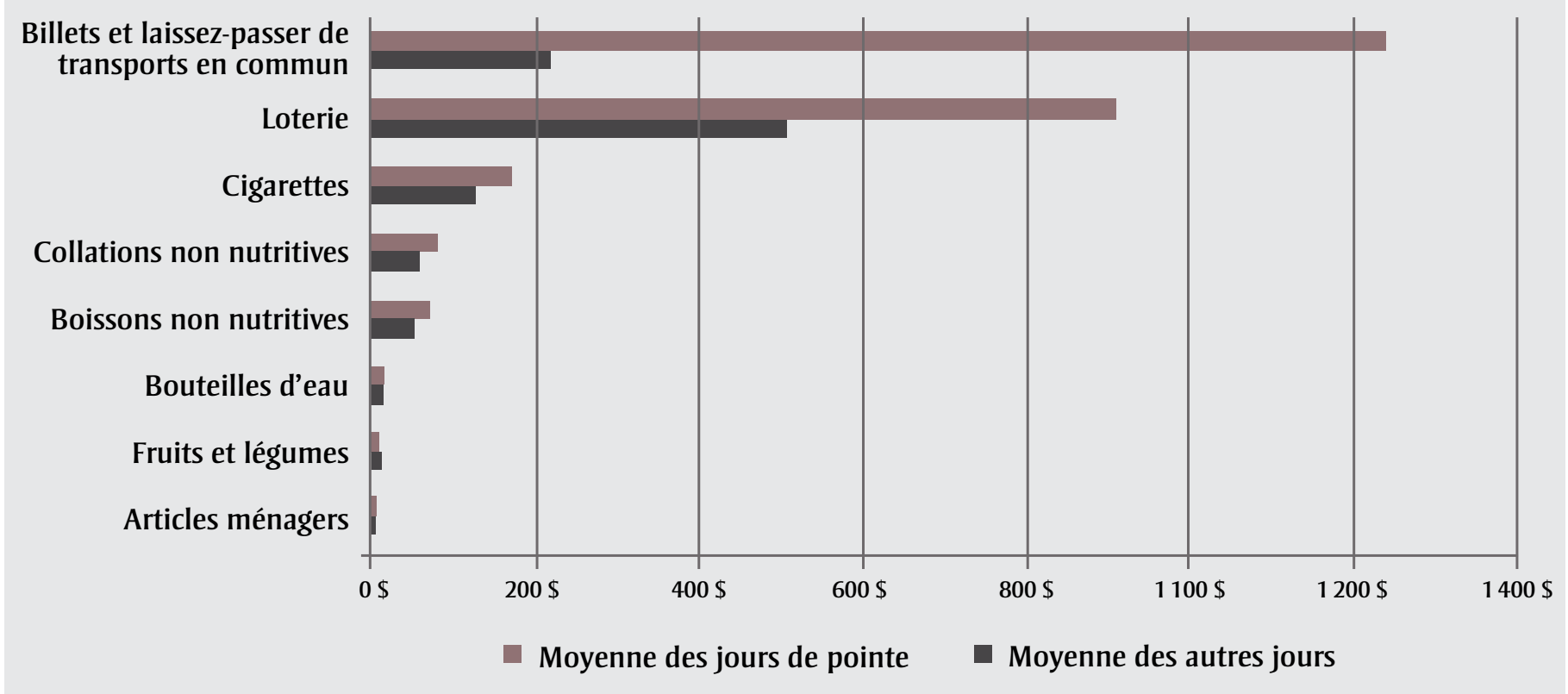

dépanneur ayant participé à une intervention gouvernementale axée sur les dépanneurs santé. Ce dépanneur était situé dans un complexe de tours d'habitations d'un quartier à faible revenu de Toronto (Ontario).
Les données présentées sont parmi les premières données sur les ventes à être analysées dans le cadre d'une étude sur les interventions ciblant l'environnement alimentaire de la vente au détail en contexte canadien $^{10,19}$, et les premières données sur les ventes à être étudiées dans le cadre d'une intervention sur les dépanneurs santé au Canada. Cette étude ne visait pas

à commenter les répercussions au niveau

\section{FIGURE 3}

Revenu brut mensuel généré par quatre catégories de produits au cours d'une intervention ciblant un dépanneur santé de Toronto, Ontario, août 2014 à avril 2015

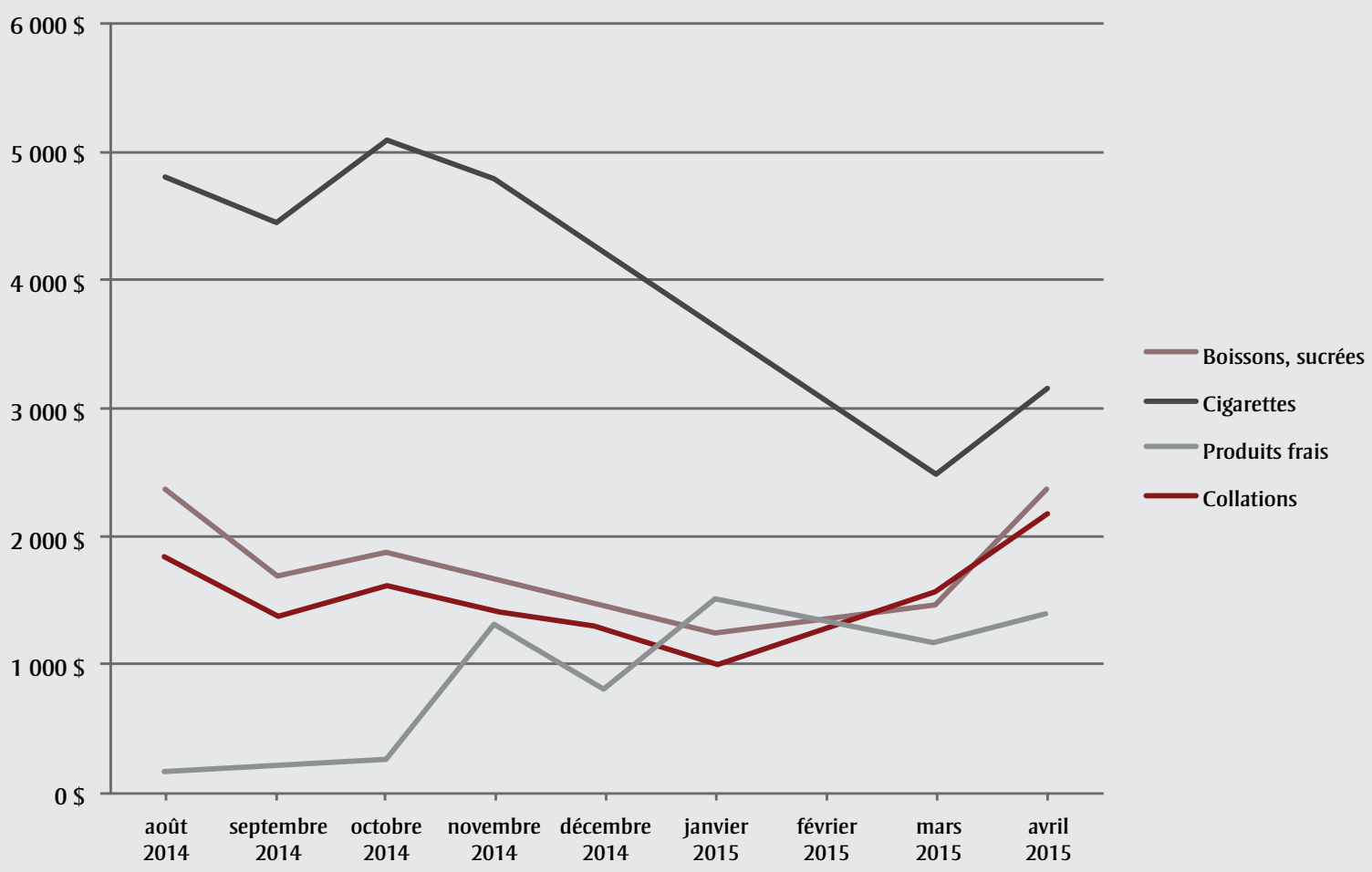

Remarque : Les collations comprennent des friandises, du chocolat, des croustilles et d'autres collations salées, ainsi que des collations à base de viande. 
FIGURE 4

Revenu brut mensuel généré par la vente de denrées fraîches lors d'une intervention ciblant un dépanneur santé de Toronto (Ontario), août 2014 à avril 2015

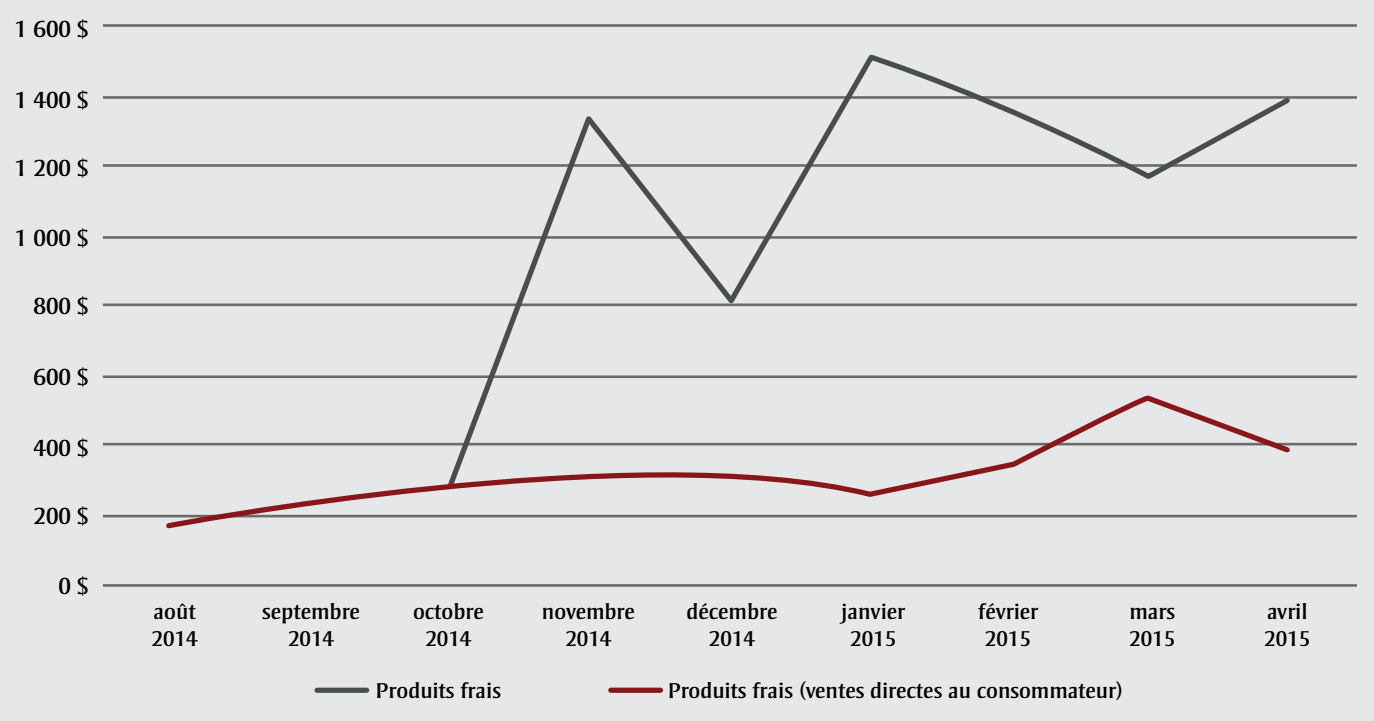

des consommateurs, mais plutôt à mettre en lumière les caractéristiques des revenus en magasin réalisés au cours d'une période d'intervention ainsi que diverses interprétations possibles des tendances liées à la vente de produits au détail dans de petits commerces urbains.

Trois constatations principales pertinentes pour la recherche et la pratique en santé publique au Canada ressortent de notre étude. D’abord, les ventes en magasin réalisées tout au long de l'intervention ont culminé à des intervalles de temps prévisibles (le jour suivant le versement des chèques d'assistance sociale). Ensuite, la moyenne des ventes de certaines catégories de produits lors des jours de ventes de pointe différait significativement de la moyenne des ventes des autres jours du mois. Les ventes en magasin recensées au cours de l'intervention ont permis d'observer un changement longitudinal dans la combinaison de produits au fil du temps. Enfin, les rapports produits et leur examen de concert avec les propriétaires pour en interpréter les résultats ont permis de promouvoir le concept de processus décisionnel fondé sur les données et semblent avoir suscité chez les propriétaires du dépanneur la volonté de vendre de nouveaux produits comme des fruits et des légumes frais.

Chacune de ces constatations est abordée plus en détail ci-dessous.
Dans un premier temps, il s'agit de la première étude, à notre connaissance, à faire état de la manière dont les ventes d'un dépanneur culminent le jour suivant le versement des chèques d'assistance sociale aux habitants d'un quartier à faible revenu. Cette constatation a une incidence sur les interventions à effectuer dans les dépanneurs de quartiers similaires. Plus précisément, les stratégies incitant les consommateurs à modifier leurs comportements liés à l'achat d'aliments lors des jours de ventes de pointe (quand l'affluence dans le magasin est susceptible d'être élevée), les interventions en magasin, entre autres, pourraient être explorées. La part des ventes totales du magasin consacrées aux billets de loterie, aux billets de transports en commun et aux cigarettes ainsi qu'aux collations et boissons non nutritives était significativement ou légèrement plus élevée les jours de ventes de pointe, mais aucune différence n'a été notée dans la vente de fruits et légumes, d'eau en bouteille ou d'articles ménagers. Ces constatations mettent aussi en lumière l'importance de distinguer l'analyse de données sur les ventes à une échelle globale (magasin ou collectivité) de celle effectuée à l'échelle de l'individu. La part des ventes totales du magasin provenant de diverses catégories de produits ne peut pas être attribuée aux caractéristiques des individus ou des ménages. Cependant, des recherches antérieures réalisées auprès de ménages à faible revenu de Toronto ont révélé la débrouillardise des personnes aux prises avec des contraintes économiques graves comme une insécurité alimentaire du ménage ${ }^{25,26}$ et ont également fait état du large éventail de facteurs contribuant à la prise de décisions en en matière de budget des ménages. Par exemple, parmi les ménages à faible revenu, même si le prix des aliments constitue un facteur clé dans la prise de décision concernant les achats, et si les prestations d'assistance sociale semblent déterminer les achats mensuels d'aliments, la manière dont les ménages économisent dépend des préférences et de facteurs relatifs à la santé, de même que du caractère durable et périssable des aliments et de la valeur qui leur est attribuée ${ }^{25}$. Les recherches futures devraient explorer davantage les raisons à ces différences dans les achats alimentaires au cours d'un mois en tenant compte des contraintes économiques plus ou moins grandes selon les moments du mois.

Dans un deuxième temps, sur le plan de la mixité des produits, l'utilisation de données sur les ventes nous a permis d'analyser la variation longitudinale des ventes de différentes catégories de produits au fil du temps. Dans les magasins de détail, la majorité des ventes proviennent généralement d'un nombre restreint de gammes de produits, même si leur inventaire est diversifié. L'intervention axée sur les dépanneurs santé portait principalement sur les gammes de produits vendus 
généralement dans les dépanneurs, en particulier le tabac, les billets de loterie, les laissez-passer d'autobus et de métro, les boissons sucrées et les collations à faible valeur nutritive. Au fil du temps, toutefois, à la suite de l'intervention, le fait de faire la promotion de fruits et légumes variés et de les inclure dans l'inventaire a fait en sorte qu'ils occupent une plus grande part des revenus du magasin. Modifier la gamme de produits offerts dans les dépanneurs pour qu'elle soit plus santé requiert des qualités entrepreneuriales et créatives, comme nous l'avons souligné ailleurs ${ }^{27}$. Cette étude a mis l'accent sur les ventes en magasin, mais notons qu'une contribution importante aux revenus - $68 \%$ du total des revenus générés par les fruits et légumes provenaient des ventes effectuées à la compagnie de location immobilière propriétaire de la tour d'habitations, comme nous l'avons vu. Si l'on ne tient pas compte de cette opportunité spécifique, les ventes de fruits et légumes aux clients particuliers ont également augmenté de façon constante avec le temps, ce qu'il importe de noter pour les futurs programmes axés sur les dépanneurs santé n'offrant pas de ventes en vrac. Cela dit, d'un point de vue pratique, il semble que le succès des interventions dans le secteur de l'alimentation au détail repose sur la mise en place de solutions créatives visant la viabilité financière de ces interventions ${ }^{20,28}$. En outre, dans la mesure où les interventions axées sur les petits commerces tiennent compte d'autres objectifs en matière de santé publique (par exemple, réduire la consommation de tabac), des stratégies novatrices pour attirer la clientèle et diversifier les sources de revenus sont nécessaires.

Dans un troisième temps, la transmission des données sur les ventes aux propriétaires en vue de les analyser et de les interpréter avec eux a contribué à promouvoir le concept de processus décisionnel fondé sur les données et semble avoir suscité chez les propriétaires du dépanneur la volonté de vendre de nouveaux produits comme des fruits et des légumes frais. En utilisant les données sur les ventes, les propriétaires ont été en mesure d'évaluer objectivement les revenus générés par différentes catégories de produits et de noter quels types de marchandisages faisaient augmenter le plus les ventes de divers aliments nutritifs, et les moments où cela s'est produit. En outre, la préparation d'un sommaire des données sur les ventes avant la mise en œuvre d'une intervention pourrait fournir une idée des risques éventuels auxquels les magasins de détail doivent faire face lorsqu'on intervient sur certaines catégories de produits ainsi que des retombées potentielles dont ils pourraient bénéficier, ce qui pourrait aider à guider la mise en place de l'intervention.

\section{Points forts et limites}

L'un des points forts de cette étude est l'utilisation de données sur les ventes plutôt que de perceptions des propriétaires concernant les ventes en magasins, ce qui est couramment pris en compte dans la littérature ${ }^{2,29}$. De plus, ces résultats contribuent à enrichir la littérature visant à explorer d'autres paramètres sur les résultats d'interventions en matière d'environnement alimentaire dans la vente au détail, ces paramètres, et en particulier les données sur les ventes, n'étant traditionnellement pas pris en compte dans les recherches similaires (pour diverses raisons).

Notre étude comporte aussi plusieurs limites. Premièrement, nous avons examiné les données sur les ventes d'un seul magasin. Même si cette démarche est fréquemment employée dans la littérature à ce sujet ${ }^{2,30}$, nos résultats pourraient ne pas s'appliquer à d'autres lieux ou contextes au Canada.

Deuxièmement, nous n'avons pas été en mesure de faire état des données sur les ventes depuis le tout début de l'intervention étant donné les difficultés rencontrées concernant l'utilisation uniforme du système de point de vente lors des premiers mois. L'utilisation technique du système de point de vente a constitué un problème, mais nos conversations avec les propriétaires du dépanneur ont révélé que le manque d'uniformité dans la collecte des données au cours des premiers mois était aussi attribuable aux pratiques commerciales des propriétaires (p. ex. le fait de ne pas noter chaque article vendu). À cette dernière constatation s'ajoute le fait que le système de point de vente n'était équipé ni pour consigner des données sur l'emplacement des articles achetés dans le magasin (p. ex. dans les zones de plus grande affluence comme au début des rangées, ou près de la caisse enregistreuse), ni pour consigner des données sur les soldes et les articles à prix réduit. Les recherches à venir pourraient combiner des données issues de systèmes de point de vente et de diagrammes de planification (diagrammes indiquant l'emplacement des produits de détail sur les étagères en vue d'en maximiser les ventes) afin d'examiner de quelle manière les ventes de différents produits nutritifs varient selon leur emplacement.

Troisièmement, dans le cadre de cette étude, seul le chiffre d'affaires généré par différentes catégories de produits a été analysé et non le profit (revenus moins les coûts de vente des articles, incluant les investissements en capital et les taxes) n’ont pas été pris en compte. Nos données sur les ventes n'ont pas tenu compte du nombre d'articles vendus mais des ventes totales en dollars, ce qui fait que nous n'avons pas pu noter la variation en nombre de portions de fruits et légumes vendus. Néanmoins, les propriétaires du magasin ont intentionnellement fixé les prix des fruits et légumes de sorte qu'ils puissent concurrencer ceux du supermarché à bas prix à proximité. Ainsi, nous avions prévu que toute modification de prix, aussi minime soit-elle, n'aurait pas pu avoir de répercussions significatives sur les comportements des consommateurs (par exemple faire en sorte qu'ils évitent d'acheter dans le dépanneur). Ceci constitue une limite qui devrait faire l'objet de futurs travaux de recherche. Par exemple, même si les ventes de tabac constituaient une part importante des revenus du magasin tout au long de l'intervention, nos conversations avec les propriétaires du magasin ont révélé leur désir d'arrêter de vendre des produits du tabac en raison des risques pour la sécurité que cela implique (sur le plan des vols) et de la diminution des marges de profit. Par ailleurs, alors que les fruits et légumes représentaient une partie moins grande des revenus, les marges de profit qui en découlaient étaient généralement élevées ${ }^{30}$, ce qui constituait pour les propriétaires un incitatif financier à continuer à entreposer et à vendre ces articles.

Quatrièmement, outre des investissements en infrastructure (réfrigérateurs et rayonnages appropriés), la vente d'aliments frais dans un dépanneur nécessite un engagement considérable de la part des propriétaires et du personnel. Le personnel doit acquérir des compétences concernant la manipulation et la salubrité des aliments, l'établissement de relations avec les fournisseurs, la négociation de prix et de modalités favorables, la présentation des produits frais, la compréhension des désirs et des besoins de la clientèle et la recherche de flux de rentrées afin de réduire les 
coûts liés au gaspillage (par exemple, faire des sandwichs roulés en utilisant des légumes frais non vendus ou aménager une section pour faire des boissons fouettées avec les fruits non vendus). De plus, les propriétaires de magasin sont souvent d'avis que les produits frais ne se vendent pas, perception probablement fondée sur des expériences antérieures ${ }^{20,30}$. Les interventions ciblant les petits commerces peuvent accroître considérablement les revenus générés par les ventes de produits frais, et ainsi demeurer une intervention importante en santé de la population pour les recherches à venir ${ }^{2,30}$.

\section{Conclusion}

Les interventions dans l'environnement alimentaire de la vente au détail suscitent de plus en plus d'intérêt parmi les interventions en santé publique, étant donné les multiples résultats positifs potentiels pour la société en cas d'adoption plus large, notamment l'amélioration de l'alimentation à l'échelle de la population. De nombreuses recherches doivent être menées afin de décrire les paramètres assurant le succès de ces types d'interventions pour divers publics, en respectant les priorités des intervenants. Les données sur les ventes sont essentielles dans l'évaluation de la faisabilité économique pour les commerces adaptant leur modèle d'affaires afin d'encourager la saine alimentation au Canada, mais souvent ces données sont privées et leur collecte aux fins de recherche en santé publique exige des partenariats entre l'industrie et les chercheurs du secteur public. Ces partenariats publicprivé constituent une question litigieuse courante dans le secteur de la santé publique au Canada, mais des outils sont disponibles pour aider les acteurs en santé publique responsables d'établir des partenariats liés à l'alimentation ${ }^{31}$. Les responsables des recherches et des interventions à venir gagneraient à envisager sérieusement l'établissement de partenariats et l'inclusion de paramètres essentiels à la réussite des interventions dans le secteur de l'alimentation au détail dans divers contextes canadiens.

\section{Remerciements}

Nous tenons à souligner le travail des évaluateurs qui nous ont donné d'excellentes suggestions à propos des versions antérieures du manuscrit. LMM tient également à remercier la Société canadienne du cancer pour son aide financière sous la forme d'une bourse de perfectionnement professionnel dans la prévention du cancer (n 704744). Notre recherche a été financée par l'Agence de la santé publique du Canada.

\section{Conflits d'intérêts}

Les auteurs déclarent n'avoir aucun conflit d'intérêts.

\section{Contributions des auteurs}

LMM a conçu l'étude et a coécrit le manuscrit avec ML. BEC a réalisé les analyses de données. LMM, BEC et CLM ont élaboré et mis en œuvre l'étude FRESH principale. Tous les auteurs ont contribué à l'analyse approfondie des données, à l'interprétation des résultats, à la révision critique du manuscrit et à l'approbation de la version finale.

\section{Références}

1. Gittelsohn J, Lee-Kwan SH, Batorsky B. Community-based interventions in prepared-food sources: a systematic review [Internet]. Prev Chronic Dis. 2013;10:130073. En ligne à : http:// dx.doi.org/10.5888/pcd10.130073

2. Gittelsohn J, Rowan M, Gadhoke P. Interventions in small food stores to change the food environment, improve diet, and reduce risk of chronic disease [Internet]. Prev Chronic Dis. 2012;9:110015. En ligne à : http:// dx.doi.org/10.5888/pcd9.110015

3. Glanz K, Bader MDM, Iyer S. Retail grocery store marketing strategies and obesity: an integrative review. Am J Prev Med. 2012;42(5):503-512.

4. Cameron AJ, Charlton E, Ngan WW, Sacks G. A systematic review of the effectiveness of supermarket-based interventions involving product, promotion, or place on the healthiness of consumer purchases. Current Nutrition Reports. 2016;5(3):129-138.

5. Glanz K, Sallis JF, Saelens BE, Frank LD. Healthy nutrition environments: concepts and measures. Am J Health Promot. 2005;19(5):330-333.

6. Ni Mhurchu C, Vandevijvere $\mathrm{S}$, Waterlander W, et al. Monitoring the availability of healthy and unhealthy foods and non-alcoholic beverages in community and consumer retail food environments globally. Obes Rev. 2013;14(Suppl 1):108-119.
7. Statistique Canada. Dépenses alimentaires moyennes des ménages, par province (Canada) [Internet]. Ottawa (Ont.) : Statistique Canada; 2014 [consulté le 5 déc. 2014]. En ligne à : http://www.statcan.gc.ca/tables -tableaux/sum-som/102/cst01/famil132a -fra.htm

8. Brimblecombe J, Liddle R, O’Dea K. Use of point-of-sale data to assess food and nutrient quality in remote stores. Public Health Nutr. 2013;16(7):11591167.

9. Foster GD, Karpyn A, Wojtanowski $\mathrm{AC}$, et al. Placement and promotion strategies to increase sales of healthier products in supermarkets in lowincome, ethnically diverse neighborhoods: a randomized controlled trial. Am J Clin Nutr. 2014;99(6):1359-1368.

10. Fuller D, Engler-Stringer R, Muhajarine $\mathrm{N}$. Examining food purchasing patterns from sales data at a full-service grocery store intervention in a former food desert. Prev Med Rep. 2015;2: 164-169.

11. Ni Mhurchu C, Vandevijvere $\mathrm{S}$, Waterlander W, et al. Monitoring the availability of healthy and unhealthy foods and non-alcoholic beverages in community and consumer retail food environments globally. Obes Rev. 2013;14(S1):108-119.

12. Song HJ, Gittelsohn J, Kim M, Suratkar S, Sharma S, Anliker J. A corner store intervention in a low-income urban community is associated with increased availability and sales of some healthy foods. Public Health Nutr. 2009;12(11):2060-2067.

13. Budd N, Cuccia A, Jeffries JK, et al. B'more healthy: retail rewards - design of a multi-level communications and pricing intervention to improve the food environment in Baltimore City [Internet]. BMC Public Health. 2015; 15:283. doi : 10.1186/s12889-015-1616-6.

14. Lent MR, Vander Veur SS, McCoy TA, et al. A randomized controlled study of a healthy corner store initiative on the purchases of urban, low-income youth. Obesity (Silver Spring). 2014; 22(12):2494-500. 
15. Ayala GX, Baquero B, Pickrel JL, et al. A store-based intervention to increase fruit and vegetable consumption: The El Valor De Nuestra Salud cluster randomized controlled trial. Contemp Clin Trials. 2015;42:228-238.

16. Brimblecombe J, Ferguson M, Liberato SC, et al. Stores Healthy Options Project in Remote Indigenous Communities (SHOP@RIC): a protocol of a randomised trial promoting healthy food and beverage purchases through price discounts and in-store nutrition education [Internet]. BMC Public Health. 2013;13:744. doi : 10.1186/1471-2458 $-13-744$.

17. Magnus A, Moodie ML, Ferguson M, Cobiac LJ, Liberato SC, Brimblecombe J. The economic feasibility of price discounts to improve diet in Australian Aboriginal remote communities. Aust N Z J Public Health. 2016;40(Suppl 1):S36-S41.

18. Holmes AS, Estabrooks PA, Davis GC, Serrano EL. Effect of a grocery store intervention on sales of nutritious foods to youth and their families. J Acad Nutr Diet. 2012;112(6):897-901.

19. Minaker LM, Olstad DL, MacKenzie G, et al. An evaluation of the impact of a restrictive retail food environment intervention in a rural community pharmacy setting [Internet]. BMC Public Health. 2016;16:586. En ligne à : http://dx.doi.org/10.1186/s12889 -016-3281-9

20. Gittelsohn J, Laska MN, Karpyn A, Klingler K, Ayala GX. Lessons learned from small store programs to increase healthy food access. Am J Health Behav. 2014;38(2):307-315.

21. Gardiner B, Blake M, Harris R, et al. Can small stores have a big impact? A qualitative evaluation of a store fruit and vegetable initiative. Health Promot J Australia. 2013;24(3):192-198.

22. Mah CL, Cook B, Rideout K, Minaker LM. Policy options for healthier retail food environments in city-regions. Can J Public Health. 2016;107(Suppl 1):eS64-eS67.
23. City of Toronto. City of Toronto ward profiles: 2011 census, Ward 43 Scarborough East [Internet]. Toronto (Ont.) : City of Toronto; 2014. En ligne à : http://www1.toronto.ca/City \%20 Of \% 20Toronto/City \% 20Planning /Wards/Files/pdf/W/Ward \% 2043 $\%$ 20Profile \% 202011.pdf

24. City of Toronto. City of Toronto ward profiles: 2011 National Household Survey, Ward 43 - Scarborough East [Internet]. Toronto (Ont.) : City of Toronto; 2014. En ligne à : http:// www 1.toronto.ca/City \% 200f \% 20 Toronto/City \%20Planning/Wards /Files /pdf/W / Ward \% 2043\% 20 NHS \% 20Profile \% 202011.pdf

25. Dachner N, Ricciuto L, Kirkpatrick SI, Tarasuk V. Food purchasing and food insecurity among low-income families in Toronto. Revue canadienne de la pratique et de la recherche en diététique. 2010;71(3):e50-e56.

26. Vozoris N, Davis B, Tarasuk V. The affordability of a nutritious diet for households on welfare in Toronto. Revue canadienne de santé publique. 2002;93(1):36-40.

27. Mah CL, Hasdell R, Soo S, Cook BE, DeMaio A, Minaker LM. Entrepreneurialism and population health interventions in the retail food environment. Health Promotion International. 2017 [sous presse].

28. Mah CL, Minaker LM, Jameson K, et al. An introduction to the healthy corner store intervention model in Canada. Revue canadienne de santé publique. 2017 [sous presse].

29. Webber CB, Sobal J, Dollahite JS. Shopping for fruits and vegetables. Food and retail qualities of importance to low-income households at the grocery store. Appetite. 2010;54(2):297-303.

30. Dunaway LF, Mundorf AR, Rose D. Fresh fruit and vegetable profitability: insights from a corner store intervention in New Orleans, Louisiana. J Hunger Environ Nutr. 2016:1-10.

31. Kraak VI. Public health and food and beverage industry engagement: a tool to assess partnership opportunities and challenges. Toronto (ON): The Healthy People and Communities Steering Committee's Multi-Sectoral Partnerships Task Group; 2014. 